\title{
Revealing the Complex Structural Intergrowth Within Ternary W-Nb-O Oxide
}

Xiaobing $\mathrm{Hu}^{1,2^{*}}$, Qian Rong ${ }^{1}$, Roberto dos Reis ${ }^{1,2}$, and Vinayak P. Dravid ${ }^{1,2}$

${ }^{1 .}$ Department of Materials Science and Engineering, Northwestern University, Evanston, US.

2. The NUANCE Center, Northwestern University, Evanston, US.

* Corresponding author: xbhu@northwestern.edu

Niobium Oxide $\left(\mathrm{Nb}_{2} \mathrm{O}_{5}\right)$ can form extensive series of ternary compounds with tungsten oxide $\left(\mathrm{WO}_{3}\right)$ by means of solid-state reaction. Although many kinds of compounds such as $\mathrm{WNb}_{12} \mathrm{O}_{33}, \mathrm{~W}_{3} \mathrm{Nb}_{14} \mathrm{O}_{44}$, $\mathrm{W}_{5} \mathrm{Nb}_{16} \mathrm{O}_{55}$ and $\mathrm{W}_{8} \mathrm{Nb}_{18} \mathrm{O}_{69}$ have been identified more than 60 years ago [1, 2], only recently, it has been found that $\mathrm{W}_{5} \mathrm{Nb}_{16} \mathrm{O}_{55}$ can intercalate large quantities of $\mathrm{Li}$ at a higher charge/discharge rate. This process occurs even when the size of the tungsten niobium oxides are in micrometer scale [3], which makes $\mathrm{W}_{5} \mathrm{Nb}_{16} \mathrm{O}_{55}$ oxide a promising material for application as electrodes in fast-charging devices and all-solidstate energy storage systems. However, literature lacks of deep structural investigations of these complex oxides and only provide very low spatial resolution information.

Here, we use advanced TEM characterization to reveal the intrinsic microstructural features of $\mathrm{W}_{5} \mathrm{Nb}_{16} \mathrm{O}_{55}$ and correlate them to the fast ionic diffusion. $\mathrm{W}_{5} \mathrm{Nb}_{16} \mathrm{O}_{55}$ oxide has a monoclinic lattice with the space group of $C 2$ and lattice parameters of $a=29.8 A, b=3.8 \AA, c=23.1 \AA, \beta=126.5^{\circ}$. The structure is composed of subunits of corner-shared octahedra arranged into $\mathrm{ReO}_{3}$-like blocks that are four octahedra wide by five octahedra long (green and brown polyhedra in Fig. 1a). At the junctions between green and brown polyhedra, octahedra share the edges. The purple and brown octahedra have a relative shift of $1.9 \AA$ along the projected direction. The intersections of four neighboring $4 \times 5$ block are tetrahedrally coordinated metallic atoms.

Fig. $1 \mathrm{~b}$ shows electron diffraction pattern (EDPs) corresponding to the [010] zone-axis. There are obvious streaking features along $\{001\}^{*}$ and $\{\overline{4} 02\}^{*}$ planes indicating the occurrence of large quantities of planar defects in above directions. In order to reveal the underlying microstructural features, high angle dark filed (HAADF) images were obtained as shown in Fig. 1c. The intergrowing features of two representative layers are labeled. Within the upper layer, the intergrowth of the $4 \times 5$ and $5 \times 5$ block is demonstrated. However, within the lower layer, we can see the intergrowth of the $4 \times 6$ and $5 \times 6$ block. In general, $\mathrm{W}_{5} \mathrm{Nb}_{16} \mathrm{O}_{55}$ oxide cannot be simply described by the pure $4 \times 5$ block as shown in Fig. 1a. Complex structural intergrowth always happen. The potential influence of these complex planar defects on the ionic diffusion will be discussed [4].

References:

[1] R S Roth et al., Acta. Cryst. 19 (1965), p. 26.

[2] J G Allpress et al., Acta. Cryst. B25 (1969), p. 1156.

[3] K J Griffith et al., Nature 559 (2018), p. 556.

[4] This work made use of the EPIC facility of Northwestern University's NUANCE Center, which has received support from the Soft and Hybrid Nanotechnology Experimental (SHyNE) Resource (NSF ECCS-1542205); the MRSEC program (NSF DMR-1720139) at the Materials Research Center; the International Institute for Nanotechnology (IIN); the Keck Foundation; and the State of Illinois, through the IIN. 

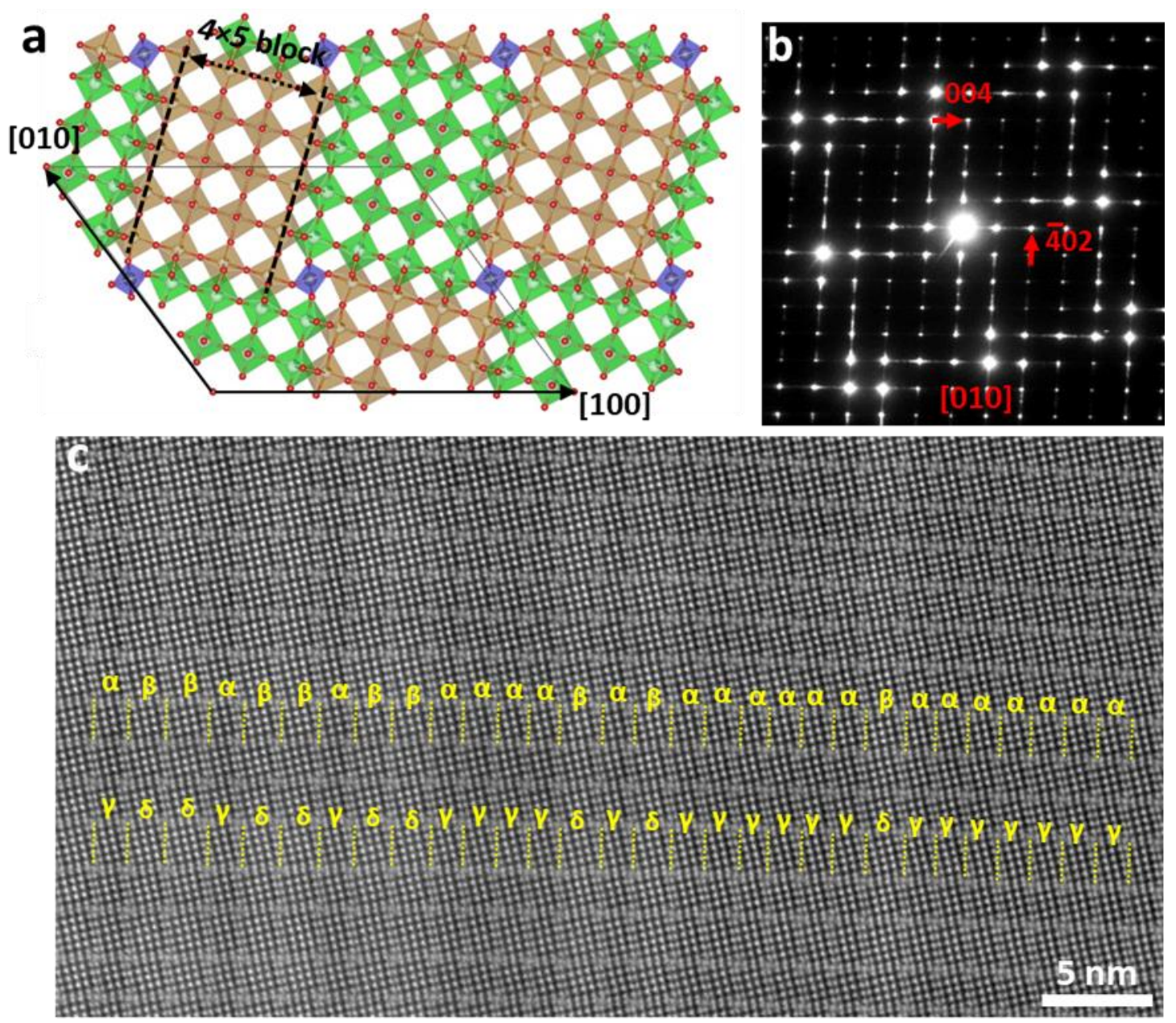

Figure 1. (a) Structural sketch of the complex $\mathrm{W}_{5} \mathrm{Nb}_{16} \mathrm{O}_{55}$ oxide projected along [010] direction. The blue polyhedra represents the tetrahedra. The brown octahedra has a relative shift of $1.9 \AA$ in contrast to the green octahedra. $4 \times 5$ block is indicated. (b) electron diffraction pattern (EDP) of $\mathrm{W}_{5} \mathrm{Nb}_{16} \mathrm{O}_{55}$ taken along [010] zone-axis. (c) Atomic resolution HAADF image showing the various structural intergrowth within $\mathrm{W}_{5} \mathrm{Nb}_{16} \mathrm{O}_{55}$ oxide. $\alpha, \beta, \gamma$ and $\delta$ denotes $4 \times 5,5 \times 5,4 \times 6$ and $5 \times 6$ block respectively. 